\title{
Caracterización del programa educación técnica y tecnológica (INATEC) en el marco del Plan Nacional de Desarrollo Humano (PNDH) de Nicaragua
}

\author{
María Elizabeth Centeno Rivera ${ }^{1}$ \\ Josseling Rodriguez Benavides ${ }^{2}$ \\ Adonis Moisés Zamora Cruz ${ }^{3}$ \\ Ethel Lizmarling Zeledón Zeledón ${ }^{4}$ \\ Beverly Castillo Herrera ${ }^{5}$
}

\section{RESUMEN}

La presente investigación desarrolla el tema de caracterización del programa de Educación Técnica y Tecnología (INATEC) en el marco del Plan Nacional de Desarrollo Humano (PNDH) de Nicaragua. Se realizó análisis documental de las teorías desarrolladas con relación al tema estudiado, así también analizamos antecedentes de un desarrollo bajo circunstancias de un contexto no progresista. Sin embargo, estas situaciones en el contexto de carencias sirvieron como factor para generar cuestiones de las necesidades de desarrollo sociales. Nos centramos en la importancia de la problemática del desarrollo y la participación ciudadana en América Latina, cabe destacar que también se trabajó con el Plan Nacional de Desarrollo Humano y el modelo Cristiano, Socialista y Solidario enfocándonos en las alternativas que posee el plan y el modelo bajo la visión de beneficiar las necesidades sociales desde la familia, comunidad y vida. La Educación Técnica y Tecnológica se ha vuelto una necesidad para desarrollar ambientes económicos y de productividad humana, dicho de otra manera, la innovación de educación y tecnología son puntos claves para visualizar un país que busca el futuro de demostrar la capacidad de educación basadas en el uso tecnológico que hoy son primordial para los crecimientos económicos y sociales de un estado.

Palabras claves: Desarrollo, participación, educación, inclusión y tecnología.

Recibido: 17 de noviembre de 2016

Aceptado: 16 de diciembre de 2016

1 UNAN-Managua/FFAREM-Estelí. Correo electrónico: elizabethcentenor10@gmail.com 2 UNAN-Managua/FFAREM-Estelí. Correo electrónico: josse_ben@hotmail.com 3 UNAN-Managua/FFAREM-Estelí. Correo electrónico: adoniszamora8@gmail.com 4 UNAN-Managua/FFAREM-Estelí. Correo electrónico: zeledonethellizmarling@yahoo.com 5 Doctora en Ciencias Sociales de la Universidad de Zulia. Docente titular de UNAN-Managua/FAREM-Esteli. Correo electrónico: beverly.castillo@yahoo.com 


\title{
Characterization of the program technical and technological education (INATEC) in the framework of the National Human Development Plan (PNDH) of Nicaragua
}

\begin{abstract}
The present investigation deals with the theme of characterization of the Program of Technical Education and Technology (INATEC) in the framework of the National Development Plan (PND) of Nicaragua. Documentary analysis was made of the theories developed with regard to the studied topic, so we also analyze antecedents of a development under circumstances of a context does not progressive. However, these situations in the context of shortcomings served as a factor to generate issues of social development needs. We focus on the importance of the issue of development and citizen participation in Latin America, it should be noted that it also worked with the National Plan for Human Development and the Christian model, Socialist and solidarity focusing on the alternatives that owns the plan and the model under the vision of benefit social needs from the family, community and life. Technical and Technological Education has become a necessity to develop economic environments and human productivity, to put it another way, the innovation of education and technology are key points to display a country you are looking for the future to demonstrate the capacity of education based on the use of technology that today are essential for the economic and social growth of a State.
\end{abstract}

Keywords: development, participation, education, inclusion and technology. 


\section{INTRODUCCIÓN}

Profundizando en la historia se pueden encontrar muchas razones que se consideran. Como el impedimento para el desarrollo de América Latina. La mala administración y la ineficiente asignación de recursos lógicamente son los responsables de la descoordinación de las políticas de desarrollo. Así también se enfatiza en las consecuencias poco positivas que tiene la dependencia muchos países con respecto a otros que en algunas ocasiones suscitan de las crisis en otras regiones, impidiendo hasta cierto punto la continuidad o el avance de procesos particulares.

La desindustrialización provoca y desencadena severos cuadros de retraso productivo y tecnológico en la economía, lo que permite que los países no disfrutan de alta niveles de crecimiento económico y bienestar. El sector primario predominante, ello significa que las exportaciones se concentran en donde las ventajas competitivas son prácticamente inexistentes. Considerando el alto volumen de exportaciones tecnológicas que existe en el comercio internacional. Otro caso particular es la mano de obra que es una considerable con niveles educativas bajo esto por supuesto es conllevado por tasas de desempleo formación profesional, pero a su vez es producto del fraccionamiento intereses nacionales. Pero también se analizaron los niveles de rentas del ahorro contribuyendo en gran parte a estimular la inversión, infiriendo de ella las consecuencias que empeora la economía.

En las próximas décadas América latina no parece salir del subdesarrollo, a pesar del financiamiento de organismos internacionales, solo países como México y Brasil que países petróleos y chile ha invertido grandemente en educación, lo que puede consolidarse como países más competitivos en la región, en el caso de nuestra región se ha ubicado muy atrás en términos de ingresos per-cápita, calidad de vida, seguridad, ciudadanía y muchos indicadores no le favorecen en su desarrollo.
Es obvio que como región debemos tecnificarnos e industrializarnos para salir de procesos y prácticas rusticas que solamente nos permite un mayor estancamiento. Es decir, también hacer un esfuerzo inmediato comunitario en comunicación con los distintos entes del gobierno, un esfuerzo para reducir la concepción ineficiencia burocracia; así como la ignorancia sumisa en la gran parte de la población sumergida en prácticas pasadas que favorecen aún más en los problemas de desarrollo de Nicaragua.

El presente artículo se enfocará en estudios de reproducción, teorías y análisis que convergen en antecedentes de América Latina y que competen a Nicaragua, los cuales son los comportamientos recientes. Así sismo permitirá mostrar los cambios de América Latina en siglos pasados y en América Latina reciente y profundizar los conocimientos teóricos en Nicaragua, así también las adaptaciones, transformaciones y alternativas a diferentes progresos.

\section{Los conceptos de desarrollo en América Latina}

En América latina existen diferentes corrientes ideológicas, críticos estudiosos y conceptos de desarrollo. A medida que pasan los años vienen surgiendo ideas concretas del bienestar común, las que están apegadas a la realidad que se vive hoy, y con un sentido más humanista tomando en cuenta las problemáticas ambientales y estructurales que posee nuestro continente.

Desarrollo en términos simplificados puede ser concebido como un proceso de transformación social orientado hacia un fin o meta. La necesidad de esta transformación puede surgir como efecto de las influencias de factores internos o externos o como resultado de la combinación de ambos factores. En todo caso, las consecuencias que resulten de este proceso tienden a gestar un nuevo orden social que implica un cambio de sistema o simplemente a mejorar la posición que se ocupa dentro del mismo. 
El enfoque de políticas públicas activas, correctamente aplicado, puede armonizar mejor con el mercado que los enfoques alternativos predominantes durante la primera fase de las reformas. En este contexto, son políticas públicas todas aquellas acciones organizadas en torno a objetivos de interés colectivo, y no solamente las estatales (CEPAL, 2000). El documento reconoce de este modo la necesidad de visualizar lo público en un sentido amplio, que involucra múltiples instancias de la sociedad civil. Esta visión de lo público encaja, además, con las necesidades de abrir espacios de participación a la sociedad civil, de avanzar en la resolución de una crisis de los Estados no plenamente superada, de corregir tanto fallas del mercado como fallas del gobierno y, más en general, de construir y reconstruir instituciones, sin duda una de las tareas más complejas que enfrenta la región.

El desarrollo sustentable es el proceso por el cual se preserva, conserva y protege solo los Recursos Naturales para el beneficio de las generaciones presentes y futuras sin tomar en cuenta las necesidades sociales, políticas ni culturales del ser humano al cual trata de llegar el desarrollo sostenible. (Machicado, 2009). La sustentabilidad debe durar en el tiempo. Compensaba hacerse sostenible y ser capaz de satisfacer las necesidades mínimas del ser humano como: alimentación, vestido, techo y empleo. En segundo lugar, también hacer sostenible otras necesidades tales como preservar la cultura, idioma, belleza del paisaje etc. (Matute, 2016)

El PNDH está concebido en el modelo cristiano, Socialista y Solidario y prioriza a los desposeídos, para caminar a la par de la justicia, de la igualdad, es un modelo, un proyecto ético en todas sus extensiones, que está puesto en práctica con una forma justa para redistribuir no sólo los recursos, sino las oportunidades y capacidades para el desarrollo pleno de las funciones humanas fundamentales de toda la población nicaragüense. (GRUN, 2014)
El presente artículo tiene como objetivo caracterizar el Programa Educación Técnica y Tecnológica INATEC enmarcado en el enfoque de desarrollo y participación ciudadana definido en el Plan Nacional de Desarrollo Humano 2007-2016 de Nicaragua.

\section{MATERIAL Y MÉTODO}

Las etapas de la investigación abarcaron lo siguiente:

Investigación documental: revisión bibliográfica y documental teórico conceptual vinculado con el tema a objeto de estudio: conceptos de desarrollo, programas sociales en Latinoamérica y la experiencia del programa de INATEC. Se obtuvieron documentos en: internet, INATEC y entrevistas a dirigentes y protagonistas de dicho instituto.

Diseño y aplicación de instrumentos: Se aplicaron dos tipos de entrevistas semi estructuradas. Una entrevista se aplicó a directores y coordinadores, enfocadas en los temas de implementación Técnica y Tecnológica. Se entrevistaron a: Rigoberto Antonio Olivas (director), Seneyda López Altamirano (Subdirectora), a María de los Ángeles Cepeda (Administradora) y los coordinadores Glorismar Antonio Carmona González (Metodólogo del área de Industria) y Alma Dania Valdivia (Metodóloga de Comercio y Servicio).

La segunda entrevista semi estructurada se les realizó a 10 protagonistas, la cual se dividió en dos bloques: 5 a los estudiantes de Computación Técnica y las otras 5 a estudiantes de Técnico Mecánico Automotriz, sus nombres se quedaron en el anonimato por seguridad de ellos o por la confianza de darnos respuestas a las 9 preguntas, estas consistían en los temas de participación ciudadana.

Se aplicó la observación directa en el Instituto la organización y estructuración de los diferentes programas, cursos y proyectos del INATEC. 
Elaboración del documento final: la información recopilada se analizó y organizó a partir de una guía facilitada por la docente de la clase de Sociología del Desarrollo.

\section{DESARROLLO}

\section{Modelo Cristiano, Socialista y Solidario (PNDH) ${ }^{1}$}

La historia de Nicaragua está plagada de guerras, golpes de estado, dictaduras. Sin embargo, es una tierra donde las mujeres y hombres han dado la vida para salvaguardar la soberanía y los derechos de los ciudadanos. En Nicaragua desde su independencia de la corona ha existido ansias de poder, partidos políticos sin fundamentos, líderes populistas que eran manipulados por los Estados unidos, sus políticas neoliberales dejaron al país con deudas casi imposibles de pagar y una sociedad sin educación y con grandes índices de pobreza.

Las recientes elecciones, ganadas inobjetablemente por el FSLN, evidenciaron la existencia de dos modelos encontrados, dos propuestas diferentes, dos proyectos distintos en Nicaragua. Nos referimos a las diferencias entre lo que propone y practica el modelo políticosociocultural que impulsa el FSLN y el decadente sistema que los partidos de derecha han promovido en nuestras latitudes.

El Sandinismo, dirigido por nuestro comandante Daniel Ortega Saavedra y la compañera Rosario Murillo. En lo que implica a nuestro país, ha promovido un modelo liberador, promotor, impulsor no sólo de las potencialidades de la ciudadanía, sino un modelo que siembra determinados comportamientos socioeconómicos que van tras el encuentro con el bien común, la colectividad, la justa re-distribución. De ahí los impactos en el mejoramiento de las condiciones de

1 Midence, Carlos (2011). NICARAGUA: Características socioeconómicas del modelo sandinista: desarrollo con justicia social. URL: http://tortillaconsal.com/tortilla/en/node/10390 vida de miles de familias que, durante el neoliberalismo, habían sido abandonadas.

En el modelo sandinista los comportamientos económicos están guiados por la interacción entre las costumbres, los hábitos culturales, las formas de producción populares y la actividad económica que debe impulsar el Estado. Se ha irradiado el cristianismo, el socialismo y la solidaridad como fundamentos sustanciales de este modelo. Es por ello que el enfoque está basado en el humano, entendiéndolo, no sólo en su dimensión biológica, sino en su realidad social, cultural y psicológica. Es lo que se ha llamado enfoque desde el desarrollo humano. El modelo sandinista es la alternativa al modelo arcaico, fracasado, elitista, colonialista del neoliberalismo.

Al asumir el Gobierno de Reconciliación y Unidad Nacional se estableció un dialogo permanente con diferentes sectores del País ejercitando de esa manera la DEMOCRACIA DIRECTA del Poder Ciudadano, para que todos los nicaragüenses, independientemente de nuestros credos políticos o religiosos y nuestras condiciones sociales y económicas, tengamos de aquí al 2012 y más allá, un PLAN NACIONAL DE DESARROLLO HUMANO, que nos una en medio de la diversidad para hacer nuestra NUEVANICARAGUA que supere la pobreza, dentro del marco del nuevo modelo de desarrollo alternativo del Poder Ciudadano.

El Plan Nacional de Desarrollo Humano (PNDH) de Nicaragua tiene como objetivo operativo el crecimiento económico con aumento del trabajo y reducción de la desigualdad y la pobreza. Los resultados 2007-2010 destacan una reducción significativa de la desigualdad entre los nicaragüenses por razón de una mejor distribución del ingreso y el consumo, que se traduce en mejores condiciones de vida de la población, especialmente de los más pobres. Esto ha sido posible por políticas gubernamentales redistributivas con resultados positivos, por una recuperación y crecimiento económico positivo en medio de una 
crisis financiera y económica mundial, por un clima de confianza que ha llevado a mayores niveles de cohesión social y alianzas nacionales que dan estabilidad para el desarrollo productivo y el aumento de las inversiones, entre otros factores que combinados obtuvieron este fin.

En 2009 comparado a 2005, Nicaragua ha logrado una reducción en pobreza general de 5.8 puntos porcentuales y 2.6 puntos en pobreza extrema. Los principales instrumentos utilizados para reducir la desigualdad y la pobreza en el período 2007- 2010 han sido las políticas sociales dirigidas para fortalecer las capacidades de los más pobres y contribuir a mejorar su nivel de vida. Se restituyó los derechos humanos constitucionales de salud y educación gratuitas. Se trabajó en dar la batalla en 2012 y el año en 2015; se implementaron programas sociales focalizados como: programa AMOR, programa AMOR para los Más Chiquitos, programa de Salud Familiar y Comunitario (MOSAFC), Operación Milagro y el programa Todos con Voz.

Se ha capitalizado a los pobres como agentes del desarrollo con énfasis en la participación social y económica de las mujeres contribuyendo a la nutrición, seguridad alimentaria y generación de trabajo e ingresos a través de programas como Hambre Cero y Usura Cero. Se ha apoyado a la pequeña producción urbana y rural con financiamiento, insumos y asistencia técnica con énfasis en los pequeños productores que son el 94 por ciento del total de productores. Estos productores producen el $60 \%$ del café y carne, los dos principales productos de exportación de Nicaragua, 80 por ciento de los granos básicos y 90 por ciento de las frutas y hortalizas. Además, generan, junto con la pequeña producción urbana, 70 por ciento del trabajo y 40 por ciento del PIB.

\section{PROGRAMA DE EDUCACIÓN TÉCNICA Y TECNOLÓGICA (INATEC)}

\section{Antecedentes del programa Educación técnica y tecnológica $^{2}$}

La Educación Técnica en Nicaragua se inició en 1951 mediante un convenio de asistencia técnica y ayuda económica, firmada por los gobiernos de Estados Unidos y Nicaragua con la finalidad de satisfacer la demanda de técnicos que la industria nacional requería para su desarrollo.

Los primeros centros creados fueron: Instituto Técnico Vocacional fundado en 1951 en la ciudad de Managua, Instituto Tecnológico de Granada (INTECNA) fundado en 1969, Instituto Técnico La Salle de León, fundado en 1970, Centro Juvenil Don Bosco de Managua en 1970, Instituto Técnico Cristóbal Colón de Bluefields en 1972. Las especialidades que se ofertaban en estos centros eran: Electricidad, Soldadura, Metal Laminado, Refrigeración, Aire Acondicionado, Radio y Televisión, Mecánica Automotriz y Mecánica de Torno. En las décadas del 70 al 90 llegaron a conformarse 47 centros técnicos entre Institutos, Centros Básicos de Producción (CBP) y Escuelas Campesinas que ofertaban 30 especialidades en los niveles de Técnico Básico y Técnico Medio para los tres sectores de la economía nacional, formación que era rectorada por el Ministerio de Educación.

La capacitación por su parte tuvo su origen en el Instituto Nacional de Aprendizaje (INA), organismo creado en 1967 bajo la dirección del Ministerio del Trabajo y asesoría de la Organización Internacional del Trabajo (OIT). En 1979 el INA experimentó cambio en su funcionamiento y se constituyó en el Sistema Nacional de Formación Profesional (SINAFOR), bajo cuya estructura comenzó operar en mayo de 1980, lo

2 GRUN (2012). Plan Nacional De Desarrollo Humano 2012-2016. Versión preliminar de consulta nacional. Managua, Nicaragua. URL: http://www.pndh.gob.ni/documentos/pndhactualizado/ pndh.pdf 
que conllevó a una ampliación de sus instalaciones y extensión de su cobertura a los tres sectores de la economía. En 1986 el SINAFORP fue reestructurado y pasó a llamarse Sistema Nacional de Capacitación (SINACAP), se le otorgó autonomía y asignó la función rectora de todas las acciones de capacitación. En 1988 el SINACAP fue adscrito al Ministerio de Educación y dos años más tarde pasó bajo la dirección del Ministerio del Trabajo.

Los recursos y esfuerzos de la Educación Técnica y Capacitación, que hasta fines de 1990 se encontraban dispersos en los diversos Centros Técnicos y de Capacitación, se unificaron en una sola institución, al crearse en 1991 el Instituto Nacional Tecnológico (INATEC) como ente rector de la formación profesional en Nicaragua.

El Instituto Nacional Tecnológico (INATEC), es una institución autónoma con personalidad jurídica $\mathrm{y}$ patrimonio propio, creado por decreto Presidencial No. 3-91 de enero 10 de 1991 y decreto No 40-94 "Ley Orgánica“, para constituirse en rector de la formación profesional en Nicaragua y contribuir al desarrollo económico-social del país mediante la educación técnica y capacitación de los recursos humanos calificados que demandan los sectores productivos.

El Instituto Nacional Tecnológico -INATEC-, desde su creación en 1991 es el ente rector y formador de la Formación Profesional de Nicaragua, constituyéndose desde entonces comopilarfundamentalen la preparación de los recursos humanos calificados necesarios para el logro de los objetivos de crecimiento económico y desarrollo social de nuestro país. Para el INATEC constituye una prioridad capacitar técnicamente a personas con problemas de desempleo, subempleo y las que viven en pobreza y pobreza extrema a causa de una baja o ninguna calificación. También ejecuta con carácter de prioridad programas especiales con componentes de capacitación y asistencia técnica para mujeres desempleadas, microempresarios(as) y personas con capacidades diferentes y un Programa de Capacitación en Autoconstrucción de Viviendas para capacitar en técnicas básicas de Construcción Civil a sectores de población menos favorecidos y que además carecen de viviendas. También brindamos capacitación y/o educación técnica a trabajadores de las empresas e instituciones que aportan el $2 \%$ de su masa salarial, con el fin de aumentar su productividad y eficiencia en el desempeño de sus labores.

\section{Estelí cuenta con uno de los 43 centros tecnológicos del país ${ }^{3}$}

Se conoce como Instituto Politécnico Estelí-IPE, comandante Francisco Rivera Quintero "El Zorro". Estelí cuenta con uno del centro de educación técnica y tecnología ubicado actualmente en el $\mathrm{B}^{\circ}$ Óscar Gámez Número 2 , del tanque verde $2 \mathrm{C}$. al oeste y 1 cuadra al norte, en la parte oeste del casco urbano de la ciudad de Estelí. Fue fundado en el año 1987 y se crea como Instituto Nacional Técnico para la Administración y Economía Carlos Fonseca Amador-INTAECFA Estelí, formando parte del Sistema Nacional de CapacitaciónSINACAP y posteriormente del Instituto Nacional Tecnológico-INATEC en 1992.

En el año 2010, el Instituto Nacional Técnico para la Administración y Economía-INTAE, pasó a llamarse Instituto Politécnico Estelí-IPE, comandante Francisco Rivera Quintero “El Zorro” desde el año 2014 hasta la actualidad.

En relación al Curriculum Técnico ofertado, este ha variado, ya que al inicio la formación técnica estaba orientada al área de comercio, manteniéndose hasta 1995. A partir de 1996 se va ampliando en diferentes servicios: Hotelería, Computación, Construcción, Electricidad, Industria y Bachillerato Técnico en Contabilidad y Administración. Así mismo desde

3 Esta información es obtenida de: GRUN-Gobierno de Reconcliacion y Unidad Nacional e INATEC (2014). Antecedentes. Pág. 1-2 
el año 2012 se atienden en las empresas aportadoras al 2\% para desarrollar cursos de capacitación a sus empleados.

Desde la fundación del centro hasta la actualidad el propósito principal del IPE ha sido llegar a diversas personas de escasos recursos económicos, trabajadores empíricos en diversas áreas profesionales para facilitar su inserción laboral, autoempleo, desarrollo de competencias laborales y al desarrollo económico de los municipios del norte de Nicaragua.

Instituto Politécnico Estelí-IPE, brinda servicios de Educación Técnica y profesional en las ramas de: 1) Comercio y servicios (Administración, Contabilidad, Banca y Finanzas, Computación, Secretariado ejecutivo, Hotelería y Turismo, Cocina, Pastelería y Barman Profesional); 2) Industria (Energía Renovable y Mecánica Automotriz); 3) Cursos de habilitación (Belleza y Costura); y 4) Bachillerato Técnico.

Para la prestación de los diferentes servicios técnicos profesionales, el Instituto Politécnico Estelí-IPE, cuenta con una fuerza laboral de 39 trabajadores de tiempo completo y 116 facilitadores, para atender una matrícula entre 5,000 y 6,000 protagonistas anualmente.

\section{Políticas y estrategias organizativas del Programa} Instituto Nacional Técnico y Tecnológico (INATEC) ${ }^{4}$

POLITICA 1: Normar y fortalecer los procesos institucionales desde una perspectiva de Calidad.

Estrategia 1.1: Fortalecer el proceso de organización estructural y funcional del INATEC, estructurando un modelo de gestión participativo, articulado, transparente y flexible, en base a la Ley 290, las NTCI de la CGR y la Estrategia Educativa Nacional.

4 Esta información fue obtenida de: GRUN-Gobierno de Reconciliacion y Unidad Nacional e INATEC (2014). Plan Estratégico Institucional del INATEC. Pág. 11-17
- Adecuar la estructura organizacional del INATEC (considerando resultados del diagnóstico de funciones y cargos) que garantice el cumplimiento de la Misión Institucional, la Calidad de la gestión, adopción de principios y valores definidos en el Plan Nacional de Desarrollo Humano.

- Determinar los requerimientos y requisitos de la organización estructural y funcional para su aprobación ante el Consejo Directivo.

Estrategia:1.2: Establecer indicadores de Calidad en todos los procesos Institucionales para medir la satisfacción de los(as) usuarios(as) y la adopción de un sistema de gestión de calidad.

- Fundamentar la base para la decisión de las autoridades competentes sobre el sistema de gestión de calidad que adoptara el INATEC en Centros de Formación Profesional.

- Sistematizar los procesos internos existentes aplicando indicadores y procedimientos de calidad para la proyección al corto plazo.

Estrategia 1.3: Mejorar las condiciones tecnológicas que faciliten la medición de los resultados obtenidos de los diferentes procesos institucionales y que contribuyan a una efectiva toma de decisión.

- Fortalecer los sistemas informáticos para homologar las estadísticas y reportes de INATEC con los requerimientos de los Sistemas de Gobierno de la República en un banco de Datos Único.

- Diseñar plataformas especiales que respondan a las necesidades de las y los diferentes usuarios(as) (Plataforma de Redes de Centros, Plataforma Docentes, Plataformas Temáticas etc.) que propicien el intercambio y actualización de Información.

Estrategia 1.4: Sistematizar el proceso de la planificación del INATEC para cumplir con la Misión y los Objetivos institucionales. 
- Establecer un proceso de planificación institucional de acuerdo a los requerimientos de la Misión y Visión Institucional y del Gobierno de Nicaragua.

- Elaborar e implementar el Manual de Planificación en todos los niveles de la Institución.

Estrategia 1.5: Vincular el presupuesto a la planificación Institucional que asegure los recursos financieros para el desarrollo de proyectos de inversión en los diferentes procesos de la Formación Profesional.

- Elaborar estudios y diagnósticos tecnológicos, científicos y de infraestructura con el fin de determinar las necesidades de desarrollo Institucional

- Contribuir a la actualización y modernización tecnológica de los Centros de Formación profesional con base al avance científico y tecnológico y de las nuevas demandas de Formación.

\section{POLITICA 2: Posicionar y Consolidar la Educación} Técnica y la Capacitación como una alternativa de la población para mejorar su calidad de vida.

Estrategia 2.1: Promocionar y divulgar permanentemente la oferta de servicios de Educación Técnica y Capacitación que brinda el INATEC a los diferentes sectores económicos y sociales del país.

- Fortalecer las acciones de comunicación y divulgación, sobre las ofertas de educación técnica y capacitación como una alternativa a la población para mejorar sus niveles de vida.

- Desarrollar procesos de comunicación, para valorar el papel de la Educación Técnica y la dignificación de los oficios con los diferentes actores locales.

Estrategia 2.2: Posicionar al INATEC como el rector del subsistema de Formación Profesional y Educación Técnica.

- Garantizar la interrelación entre los diferentes subsistemas, para coordinar los mecanismos de gestión del sistema educativo nacional.

- Asegurar la Articulación entre los subsistemas educativos para que sus egresados tengan acceso a las opciones formativas que cada uno ofrece.

POLITICA 3: Contribuir al desarrollo socioeconómico del país a través de una oferta de Educación Técnica y Capacitación pertinente, flexible y de Calidad enmarcado en el nuevo modelo de participación ciudadana.

Estrategia 3.1: Vincular la oferta formativa y educativa de INATEC a la transformación productiva de sectores prioritarios, considerados en el PNDH, para el crecimiento de la economía nacional.

- Diversificar la oferta educativa enfocada al desarrollo socioeconómico local, a través de una formación profesional y técnica, pertinente, flexible y de calidad.

- Articular la Oferta de Educación técnica y Formación Profesional a la demanda de los sectores económicos y sociales en correspondencia con las potencialidades de desarrollo de las localidades y el avance tecnológico.

Estrategia 3.2: Promover la investigación, desarrollo e innovación para propiciar el mejoramiento de la oferta formativa y el proceso de enseñanza - aprendizaje vinculado con el desarrollo productivo del país.

- Articular con las diferentes instituciones formativas y tecnológicas que realizan investigaciones para mejorar y actualizar currículo, calificación del personal docente y Especialistas de la Educación técnico y Formación Profesional.

- Sistematizar el Modelo metodológico de la formación basada en competencias laborales.

Estrategia 3.3: Fortalecer las competencias técnicas y metodológicas de los(as) Docentes para brindar una educación técnica y formación profesional de Calidad. 
- Promover un programa de profesionalización en coordinación con los otros subsistemas educativos que permita ampliar y actualizar las competencias técnicas y metodológicas de los(as) docentes.

- Certificarlascompetencias técnicasymetodológicas de los y las docentes de la Educación Técnica y Formación Profesional pública y privada.

Estrategia 3.4: Mejorar la calidad de los procesos de atención a empresas y de acreditación y supervisión de los Centros de Formación Profesional públicos y privados.

- Promover el dialogo y alianzas con empresarios, micro y medianas empresas, para asegurar la calificación que nos permita más calidad $\mathrm{y}$ competitividad.

- Diseñar un modelo de supervisión integral que permite la aplicación de criterios únicos para contribuir a mejorar la calidad los procesos de Educación técnica y Formación Profesional.

Estrategia 3.5: Desarrollar acciones para la conservación del medio ambiente a nivel institucional.

- Diseñar y aplicar una campaña de sensibilización sobre buenas prácticas ambientales que contribuyan al control y la reducción de la contaminación y protección de los recursos naturales a nivel institucional.

- Participar en cruzada nacional de reforestación y acciones de reforestación en coordinación con entidades al nivel local y nacional.

Estrategia 3.6: Elaborar e implementar las prácticas de género en el quehacer institucional.

- Diseñar e implementar campañas permanentes de sensibilización en prácticas de género a nivel Institucional.

- Integrar las prácticas de género en todas las acciones $\mathrm{y}$ toma de decisiones del quehacer institucional.

\section{Participación ciudadana en el programa Educación Técnica y Tecnológica ${ }^{5}$}

El artículo 7 de la constitución política de Nicaragua establece que Nicaragua es una República democrática, participativa y representativa, así como en el artículo 50 se garantiza el derecho de la participación ciudadana en igualdad de condiciones en los asuntos públicos y la gestión estatal, a través de la ley de la materia para que norme y regule dicha participación en los asuntos nacionales y locales estableciendo el ámbito de participación y los procedimientos atingentes.

El proceso de participación ciudadana es un derecho fundamental, reconocido en la Constitución Política y en diferentes instrumentos internacionales en materia de derechos humanos que han sido ratificados por Nicaragua, tales como la Declaración Universal de Derechos Humanos, el Pacto Internacional de Derechos Civiles y Políticos y la Declaración Americana de los Derechos y Deberes del Hombre, entre otros.

El instituto nacional tecnológico en conformidad con la ley de participación ciudadana y coordinado por el gobierno de unidad nacional, brinda la oportunidad a la población nicaragüense en general, de estudiar carreras técnicas que promueve en diferentes partes del país ya que cuenta con centros de estudio en todas las regiones.

Las iniciativas de las organizaciones que involucran a las y los jóvenes, siempre van a estar bien aceptadas por la sociedad, porque es preferible que se desarrollen habilidades artísticas en estos espacios, a que estemos sumergidas en un mundo de rechazo o de adopción de un mal comportamiento o costumbres, ligadas a las adicciones y acciones vandálicas.

5 Esta información es obtenida de: Tecnológico Nacional, Innovación y prestigio 09 de junio 2015. Obtenido: http:// www.inatec.edu.ni/news/view/title:124-mejor-salud-para-losnicaraguenses-desde-el-programa-educacion-tecnica-en-elcampo 
Así que INATEC se emplea por la participación de los ciudadanos especialmente jóvenes mujeres, adultos y personas con capacidades diferentes

La región de Estelí cuenta actualmente con más de 5000 estudiantes que se distribuyen en el centro y en las escuelas de oficio en el campo con múltiples modalidades.

- En el sector agropecuario: Técnico General, Técnico Especialista y Bachillerato Técnico.

- Sector comercio y Servicio-Hotelería: Técnico General, Técnico Especialista y Bachillerato Técnico.

- Sector industria y construcción: Técnico General, Técnico Especialista y Bachillerato Técnico.

Estas son las carrearas más demandadas por la población en general, por lo que son las más promovidas por el centro. Los jóvenes se integran en todas las actividades del centro, en ferias científicas, en promoción de sus carreras para que los estudiantes de secundaria obtén por la alternativa que el Tecnológico nacional les brinda.

Para formar parte de la familia INATEC y ser partícipe de los programas y carreras técnicas que ofrece, debes tener como mínimo tercer año de secundaria aprobado, todos podemos participar sin exclusión alguna. Las modalidades que se ofrecen son de mucha demanda hoy en día en país, es por ellos que las autoridades se han propuesto para este año 2017, metas que sobrepasan las alcanzadas el año pasado.

Nuestro Gobierno de Reconciliación y Unidad Nacional Considera que la Educación Técnica y la Capacitación deben garantizar acciones articuladas, dirigidas a formar y capacitar a jóvenes y adultos del área urbana y rural que les asegure la posibilidad de un empleo decente e ingreso al mercado laboral con mejores niveles de calidad.
INATEC se encuentra activo en programas de sostenibilidad en búsqueda del desarrollo en conjunto con el gobierno, se dedicará a revisar programa por programa "tanto en términos de su rendimiento efectivo como en términos de una valoración cultural, política, sociológica de la transformación que han representado en las familias nicaragüenses todas estas acciones de lucha eficaz contra la pobreza". Se destaca el trabajo que se realiza desde sus programas donde los jóvenes estudiantes visitan y brindan asistencia técnica a familias de comunidades protagonistas del Bono Productivo Alimentario.

Con el Movimiento de Técnicos y Profesionales de la Juventud Sandinista también se está promoviendo una consulta de la estrategia nacional de dignificación de la educación técnica para adecuarla al Plan Nacional de Desarrollo del país, y con la implementación de buenas prácticas agrícola para un desarrollo integral y sostenible. A base de estiércol maduro, carbón, sácate picado, leche, harina de roca, melsas entre otros elementos orgánicos y de fácil acceso en el campo, facilitadores del programa refuerzan sus conocimientos para elaborar bíofertilizantes, abono orgánico para su correcta aplicación en los cultivos.

Son prácticas amigables con el medio ambiente que incrementa el rendimiento de las parcelas, con melaza, estiércol, levadura, cal y otros ingrediente presentes en ellas, preparando un biomineral, el que ayuda a fortalecer el crecimiento sano de las plantas, para que se desarrolle con mayor vigor, de esta forma complementamos los elementos que de manera natural no tiene la tierra; haciendo de estas prácticas un hábito en la producción agrícola, aumentaremos los niveles de rendimientos y obtendremos productos más sanos para el consumo humano.

El Instituto de Educación Técnica cuenta con la Formación Profesional dirigidas a las juventudes, los adultos hombres y mujeres, la gente del campo, las personasconcapacidadesdiferentes, los/astrabajadores/ 
as $\mathrm{y}$ pueblos originarios $\mathrm{y}$ afro descendientes. La universalización de la educación técnica y la capacitación en el mediano plazo es su elemento importante para analizar la experiencia de articulación entre la formación de los y las jóvenes, el mundo del trabajo y la formación permanente, considerando la complementariedad de infraestructuras, equipamientos y diseños curriculares. La reorientación del diseño curricular son igual las prioridades y el logro de las metas económicas y sociales del país en la modalidad de cursos móviles y de aprendizaje.

Su objetivo en sostenibilidad es la incorporación de módulos de informática e inglés técnico en los cursos de aprendizaje, a fin de mejorar las competencias para la empleabilidad de los(as) jóvenes egresados en esta modalidad priorizando especialidades de Turismo, Electrónica, Mantenimiento Industrial y Administración, Secretariado y Programación. Desarrollar en coordinación con el MINED modalidades de capacitación que brinden oportunidades de habilitación laboral, para los /as egresados/as de extra edad, los recién alfabetizados y jóvenes y adultos fuera del Sistema Educativo, a través de salidas ocupacionales con su correspondiente certificado a fin de contribuir al desarrollo local y al mejoramiento de su nivel de vida.

Se basan en universalizar la enseñanza del idioma inglés en la secundaria y la Formación Profesional garantizando la calidad en la enseñanza con una mayor frecuencia horaria en los planes de clase, medios didácticos y metodología adecuada. Para contribuir con la formación de tal manera que la metodología de la enseñanza del inglés sea efectiva y garantice que estudiantes hablen el inglés como segunda lengua. Para estos programas se cuenta con el $2 \%$ de cooperación por parte de todas las empresas y con parte del presupuesto de la Republica de Nicaragua.

\section{CONCLUSIONES}

La aportación de este trabajo consistió en abordar la problemática del desarrollo y subdesarrollo, la participación ciudadana y caracterizar la Educación Técnica y Tecnológica basándose en el Plan Nacional de Desarrollo Humano. INATEC se constituye desde entonces como pilar fundamental en la preparación de los recursos humanos calificados necesarios para el logro de los objetivos de crecimiento económico y desarrollo social de nuestro país. Constituye la prioridad de capacitar técnicamente a personas con problemas de desempleo, subempleo y las que viven en pobreza y pobreza extrema a causa de una baja o ninguna calificación. También ejecuta con carácter de prioridad programas especiales con componentes de capacitación y asistencia técnica para mujeres desempleadas, microempresarios(as) y personas con capacidades diferentes y un Programa de Capacitación en Autoconstrucción de Viviendas.

Es necesario comprender que las inserciones de nuevos programas promueven la participación ciudadana, que entre ello encontramos la financiación del gobierno de Reconciliación y Unidad Nacional (GRUN), el cual ha dado la sostenibilidad de la implementación de este programa desde sus inicios hasta la actualidad y alternativas para el futuro. El proceso de ampliación de las opciones de la gente y el nivel de bienestar que logran, se halla en el centro del concepto de desarrollo humano.

Esas opciones no son ni finitas ni estáticas pero independientemente del nivel de desarrollo, las tres opciones esenciales de la gente son: vivir una vida larga y saludable, adquirir conocimientos y tener a Cuando se asume que erradicar la pobreza es tarea de todos, es importante cambiar la visión de la pobreza como carencia que tienen muchos integrantes de la sociedad; reconociéndola como generadora de acción, pues esta última visión permite romper el círculo vicioso de la pobreza transformándolo en espirales ascendentes de 
desarrollo, ello utilizando como punto de partida las potencialidades y activos de la comunidad, reforzadas por un rol facilitador de los gobiernos en asociación concertada con los otros actores del desarrollo. Para lograr el desarrollo comunitario, es necesario contar con organizaciones de base comunitaria fortalecidas bajo los principios de democracia, equidad, permanencia, que tenga acceso a la información para hacer eficiente su gestión, que reconozca la igualdad entre hombres y mujeres y que a través de procesos de capacitación para el desarrollo construya sus capacidades para pasar de actor potencial a ser actor activo en la construcción de las ciudades. ceso a los recursos necesarios para tener un nivel decente de vida.

\section{BIBLIOGRAFÍA}

Schneider Cecilia, Welp Yanina. (2011). Orígenes y contradicciones de la participación ciudadana institucional. Revista mexicana de ciencias políticas y sociales, 48 .

¿Quienes somos? (2014). Obtenido de Tecnologico Nacional: http://www.inatec.edu.ni

Arturo, C. (s.f.). Significado PNUD. Obtenido de Significado PNUD: http://www.significados.com/ pnud/

CEPAL. (2000). Equidad, desarrollo y ciudadania. CEPAL-Naciones Unidas. Obtenido de: http://repositorio.cepal.org/bitstream/ handle/11362/2686/S2006536_es.pdf

Cornford, A. (2000). Equidad, desarrollo y ciudadanía. cepal .

Datosmacro.com. (s.f.). Nicaragua-Indice de Desarrollo Humano-IDH. Obtenido de NicaraguaIndice de Desarrollo Humano-IDH: http:/www. datosmacro.com/idh/nicaragua

Desarrollo, P. d. (14 de Diciembre de 2015). Informe sobre el Desarrollo Humano 2015. Obtenido de http://www.undp.org/content/undp/es/home/ librarypage/hdr/2015-human-development-report. html

Desarrollo., P. d. (14 de junio de 2016). Informe
America Latina y el caribe. Obtenido de http:// www.latinamerica.undp.org/content/rblac/es/ home/library/human_development/informeregional-sobre-desarrollo-humano-para-americalatina-y-e.html

Diccionario de Accion Humanitaria y Cooperacion al Desarrollo. (2005-2006). Obtenido de http://www. dicc.hegoa.ehu.es/listar/mostrar/170

Gobernanza y participacion ciudadana. (s.f.). Obtenido de http://www. participacionciudadanaenprodesarrollo.com GRUN. (2012). Plan Nacional de Desarrollo Humano. 2012-2016. Versión preliminar de consulta nacional. Gobierno de Reconciliación y Unidad Nacional - GRUN. Managua, Nicaragua: GRUN. Obtenido de http://www.pndh.gob.ni/documentos/ pndhactualizado/pndh.pdf

GRUN. (2014). Informe del presidente de la Republica al pueblo Nicaraguense y Asamblea Nacional. Managua. Obtenido de http://www.asamblea. gob.ni/wp-content/uploads/downloads/2014/05/ Informe-al-Pueblo-y-la-Asamblea-Nacional-28Mayo.pdf

Gudynas, E. (2010). La CEPAL esta atendiendo los problemas del desarrollo? Accion y Reaccion.

Machicado, J. (2009). Apuntes juridicos en la web. Obtenido de Apuntes juridicos en la web: https:// jorgemachicado.blogspot.com/2009/08/dss.html

Matute, M. I. (2016). La participación ciudadana en los proyectos de desarrollo: hegemonias $y$ disidencias un estudio de caso. Medellin, Colombia. Obtenido de http://www.bdigital.unal. edu.co/51721/1/37926172.2016.pdf.pdf

Nicaragua, P. d. (04 de marzo de 2014). Plan Nacional de Desarrollo Humano 2012-2016. Obtenido de Plan Nacional d Desarrollo Humano 2012-2016: http://www.ni.undp.org/content/nicaragua/es/ home/library/mdg/publication_1.html

Perisse, A. H. (2010). ciudadania como costruccion historica. Revista Crítica de Ciencias Sociales y Jurídicas.

Problemas de desarrollo de America Latina. (s.f.). 
Obtenido de http://www.gestiopoli.com

Programas de la Naciones Unidas para el desarrollo. (13 de Septiembre de 2016). Obtenido de https://es.wikipedia.org/wiki/Programa_de_las Naciones_Unidas_para_el_Desarrollo

Ricardo, B. (1998). Evolucion de las ideas de la CEPAL. CEPAL.

Ricardo, S. A. (2012). Estado Democratico de derecho. En S. A. Ricardo, Estado Democratico de derecho (pág. 23). San Jose: Grafica medium.
Schneider Cecilia, Welp Yanina. (2011). Orígenes y contradicciones de la participación ciudadana. Argentina: Revista Mexicana de Ciencias Políticas y Sociales.

Tecnologico Industrial Francisco Rivera "El Zorro". (2016). Obtenido de Tecnologico Industrial: www. inatec.edu.ni

Tecnologico Nacional. (2014). Obtenido de ¿Quienes somos?: http://www.inatec.edu.ni 